\title{
Intracoronary Nicorandil Relieves Multiple Coronary Vasospasm With Hemodynamic Collapse
}

\author{
Takaharu Hayashi, MD; Minoru Ichikawa, MD; Akio Iwata, MD; Tsuyoshi Nakata, MD; \\ Young-Jae Lim, MD; Masayoshi Mishima, MD
}

\begin{abstract}
A 49-year-old woman was referred to hospital because of chest discomfort. Coronary angiography revealed subtotal occlusion of the left coronary artery and the right coronary artery, but subsequent hemodynamic collapse occurred. Based on the results of intravascular ultrasound the occlusion was suspected to be caused by coronary vasospasm, which was not relieved by intracoronary injection of isosorbide dinitrate $(1 \mathrm{mg})$, but was alleviated by nicorandil $(2 \mathrm{mg})$, a potassium-channel opener. After discharge from hospital, the patient stopped taking her medication and returned complaining of chest discomfort again. Intravenous verapamil $(5 \mathrm{mg})$ did not improve it, but direct intracoronary administration of nicorandil $(2 \mathrm{mg})$ did bring relief. This case suggests that nicorandil is effective for coronary vasospasm. (Circ J 2008; 72: 327-330)
\end{abstract}

Key Words: Acute myocardial infarction; Coronary vasospasm; Negative remodeling; Nicorandil

$\mathbf{T}$ here are reports of coronary vasospasm causing acute myocardial infarction (AMI) $!^{-5}$ Nitrates and calcium antagonists are usually effective for coronary vasospasm and most patients respond to these drugs, but there are some refractory cases. Moreover, spontaneous multivessel coronary vasospasm in patients with AMI is not common, but once it occurs, there is a high rate of hemodynamic collapse. Therefore, clinicians hesitate to administer a sufficient high dose of isosorbide dinitrate (ISDN) or verapamil in this situation. There are also previous reports about refractory coronary vasospasm that was only relieved by intracoronary administration of nicorandil. In the patient presented here, multivessel coronary vasospasm caused hemodynamic collapse that was not relieved by ISDN, only by intracoronary administration of nicorandil.

\section{Case Report}

A 49-year-old woman first noted chest discomfort in early February 2006. The symptoms continued for approximately $2 \mathrm{~h}$ and occurred frequently. However, the duration became shorter, so she did not seek any treatment.

In early March, she underwent a routine examination and because the electrocardiogram (ECG) recorded at that time was abnormal, she was advised to consult a physician.

The next day, she attended hospital, and an old myocardial infarction was diagnosed from the ECG findings (Fig 1A) and her symptoms. The following day, she was transported to hospital by ambulance, complaining of chest pain. Laboratory tests revealed serum levels of total cholesterol $170 \mathrm{mg} / \mathrm{dl}$, triglycerides $56 \mathrm{mg} / \mathrm{dl}$, low-density lipo-

(Received March 11, 2007; revised manuscript received August 23, 2007; accepted September 12, 2007)

Cardiovascular Division, Kawachi General Hospital, Higashi-osaka, Japan

Mailing address: Takaharu Hayashi, MD, Cardiovascular Division, Kawachi General Hospital, 1-31 Yokomakura, Higashi-osaka 5780955, Japan. E-mail: takaharu@kawati.org

All rights are reserved to the Japanese Circulation Society. For permissions, please e-mail: cj@j-circ.or.jp protein $116 \mathrm{mg} / \mathrm{dl}$, high-density lipoprotein $51 \mathrm{mg} / \mathrm{dl}$, blood sugar $141 \mathrm{mg} / \mathrm{dl}$, and hemoglobin Alc 5.0\%. She did not have any coronary risk factors nor was she a smoker or in menopause.

Physical examination on admission revealed a heart rate of 75 beats $/ \mathrm{min}$ and blood pressure of $111 / 67 \mathrm{mmHg}$. The 12-lead ECG showed ST-segment elevation in leads II, III, and $\mathrm{aVF}$, (Fig 1B) so emergency cardiac catheterization was performed because recurrent inferior AMI was suspected. Heparin (100 U/kg body weight) was administered. Emergency coronary angiography showed subtotal occlusion of the right coronary artery (RCA; Fig 2A) and left first diagonal branch (Fig 2B). Subsequently, bradycardia occurred, her blood pressure decreased and she progressed to cardiogenic shock with hemodynamic collapse. Her blood pressure fell to $62 / 42 \mathrm{mmHg}$, and her heart rate fell to 24 beats $/ \mathrm{min}$. Accordingly an intraaortic balloon pump (IABP; Edwards Lifesciences Co Ltd, CA, USA) and temporary pacemaker (Edwards Lifesciences) were inserted. We diagnosed severe major 2-vessel disease, so IABP was essential to maintain her hemodynamics. After her vital signs stabilized, thrombectomy was performed using a 7Fr JR4 guide catheter (Launcher ${ }^{\circledR} ;$ Medtronic Co Ltd, MN, USA) and guide wire (athlete GT soft ${ }^{\circledR}$, Life Line Co Ltd, Tokyo, Japan) to cross the RCA stenosis. We aspirated thrombi with a 7Fr catheter (thrombuster II ${ }^{\circledR}$; Kaneka Co Ltd, Osaka, Japan), retrieving some white thrombi, so we administered ISDN $(1 \mathrm{mg})$ into the RCA. Because the coronary stenosis did not improve, we subsequently administered nicorandil $(2 \mathrm{mg})$ into the RCA, which relieved the subtotal occlusion. Intravascular ultrasound (IVUS) (Atlantis ${ }^{\circledR}$; Boston Scientific Co Ltd, MA, USA) was used to observe the site of stenosis in the RCA, which was a narrow vessel that seemed to show negative remodeling (Fig 3)? During IVUS, spasm occurred repeatedly, but administration of nicorandil always relieved it (Fig 4A). Angiography of the left coronary artery (LCA) showed that the first diagonal branch stenosis was also alleviated (Fig 4B).

After that, we administered nicorandil (1.3 Y ), heparin $(400 \mathrm{U} / \mathrm{h})$ and diltiazem $(0.6 \mathrm{Y})$ by continuous intravenous 
A

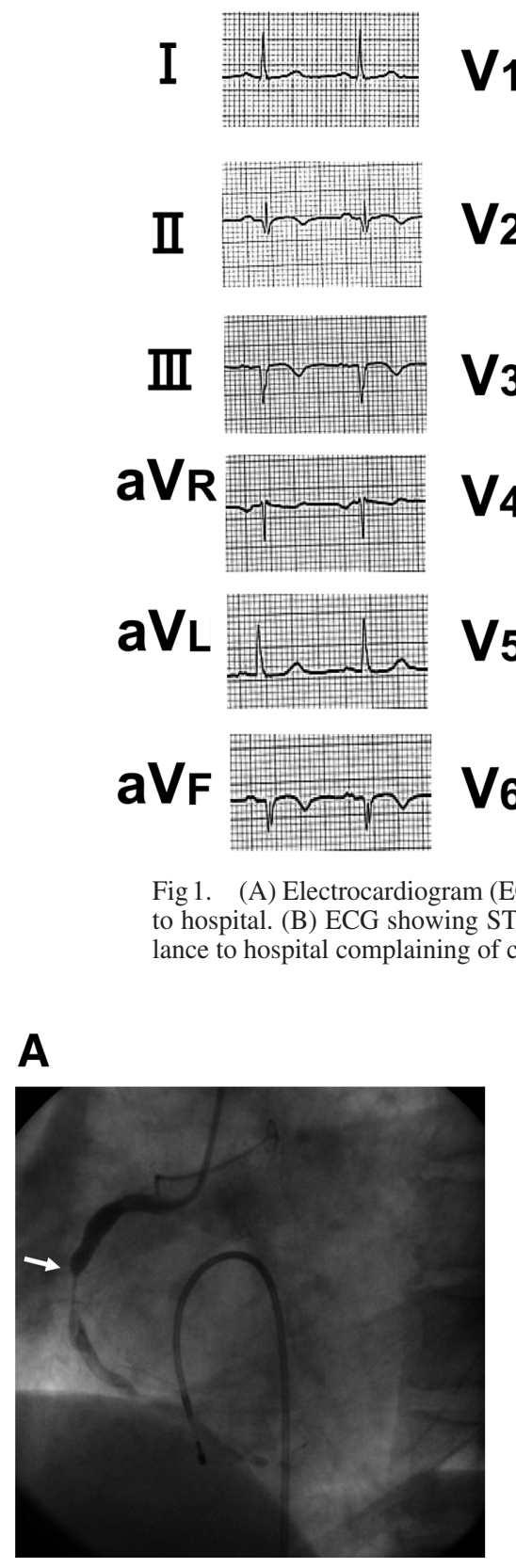

A
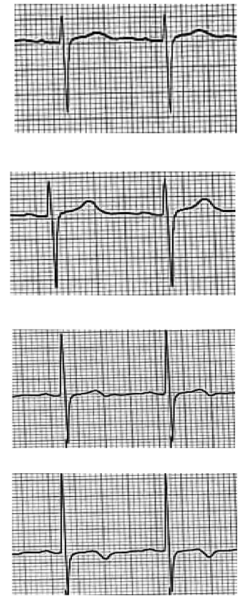

V5
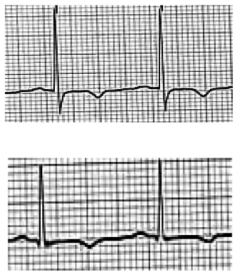

B

I

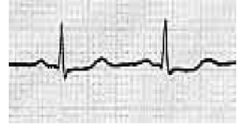

II

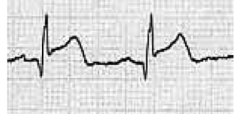

III

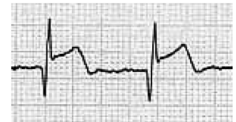

$\mathbf{a V R}$

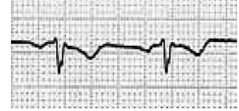

$\mathbf{a V} \mathbf{L}$

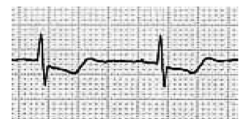

aVF

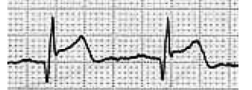

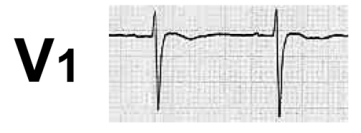
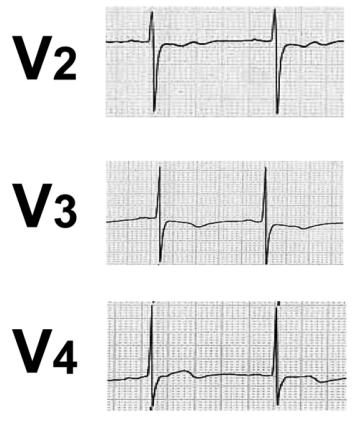

V5

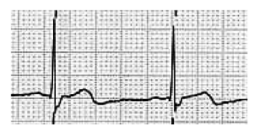

V6

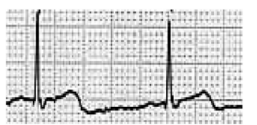

CG) showing sinus rhythm with a Qs pattern in leads II, III, and aVF on first presentation to hospital. (B) ECG showing ST-segment elevation in leads II, III, and aVF when the patient was transported by ambulance to hospital complaining of chest pain.

B

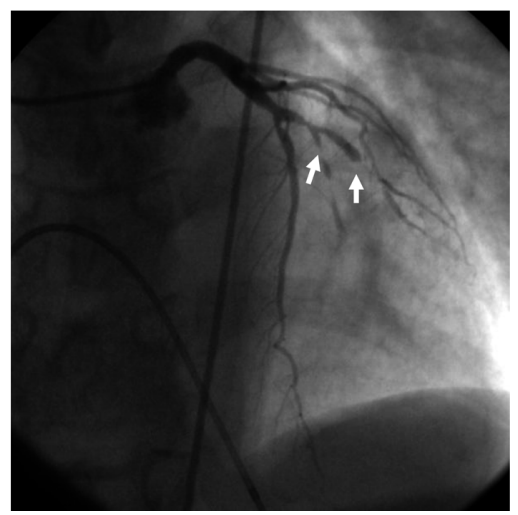

Fig 2. (A) Coronary angiography (CAG) shows subtotal occlusion with Thrombolysis In Myocardial Infarction (TIMI) grade 1 flow of the right coronary artery (arrow). (B) CAG shows subtotal occlusion, with TIMI grade 1 flow, of the first diagonal branch of the left coronary artery (arrow). infusion. Her peak serum creatine kinase level was $848 \mathrm{IU} / \mathrm{L}$ at $6 \mathrm{~h}$ after coronary intervention. Vasospasm did not recur, so we switched to oral medication, administering aspirin (100 mg/day), warfarin ( $2 \mathrm{mg} /$ day $)$, diltiazem $(200 \mathrm{mg} /$ day $)$, nicorandil (15 mg/day) and famotidine (10 mg/day).

Her symptoms remained stable, and she was discharged from hospital in early April, but 4 days later she was brought to hospital again with the complaint of chest oppression. She had stopped her medications after discharge. On the ECG, there was ST-segment elevation in leads II, III, and $\mathrm{aVF}$, suggesting the recurrence of RCA spasm. Sublingual nitroglycerin did not improve her symptoms and the ST elevation persisted in the 3 leads. Emergency cardiac catheterization revealed a new site of RCA stenosis (Fig 5A).

A 7Fr JR4 guide catheter (Launcher ${ }^{\circledR}$; Medtronic) and guide wire (athlete GT soft ${ }^{\circledR}$, Life Line) were used to cross the stenosis, but no thrombus was aspirated using a 7Fr catheter (thrombuster $\Pi^{\circledR}$; Kaneka). Therefore, we administered ISDN (1 mg) into the RCA, but the stenosis did not improve. Next, verapamil $(5 \mathrm{mg})$ was given intravenously because we considered that the stenosis was secondary to vasospasm, but there was no response, so we administered nicorandil $(2 \mathrm{mg})$ into the RCA and the stenosis was alleviated (Fig 5B).

After that, nicorandil (1.3y ), heparin (400U/h) were given by continuous intravenous infusion, then changed to oral medication again. There was no recurrence of vasospasm and she was discharged at the end of the month.

\section{Discussion}

Coronary vasospasm is reportedly common in Japanese 
A

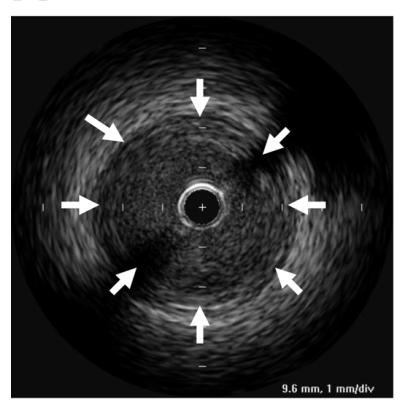

B

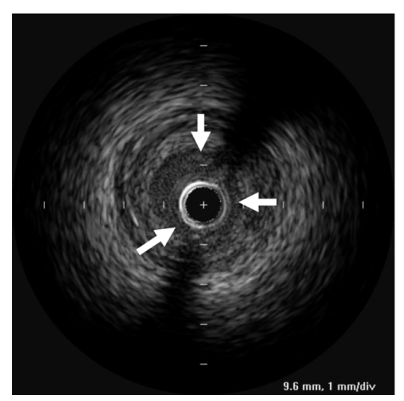

A

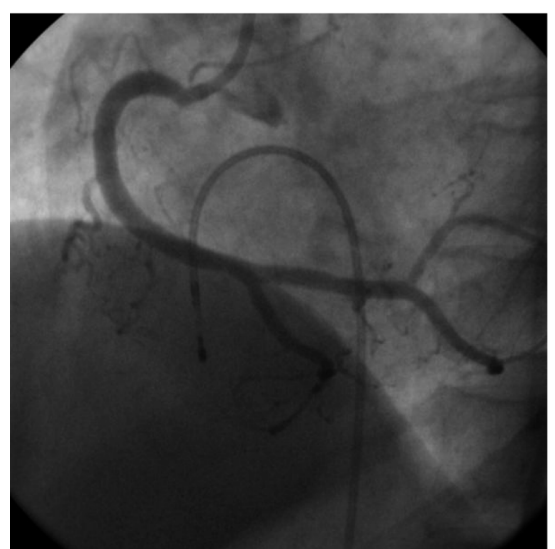

A

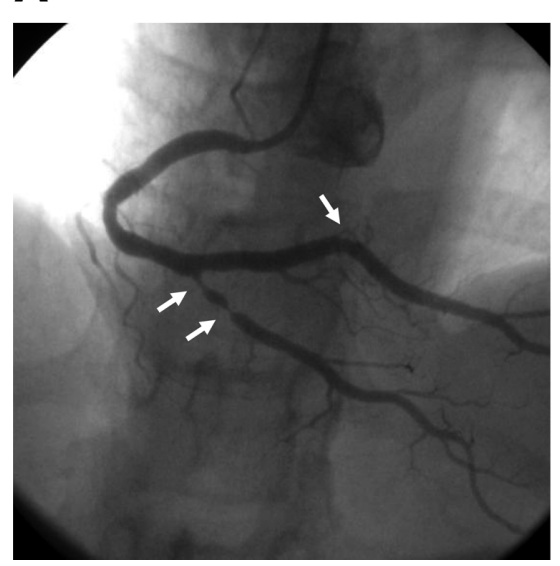

B

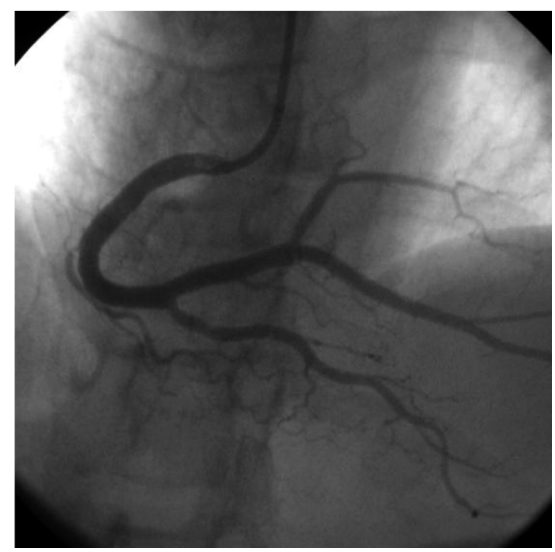

B
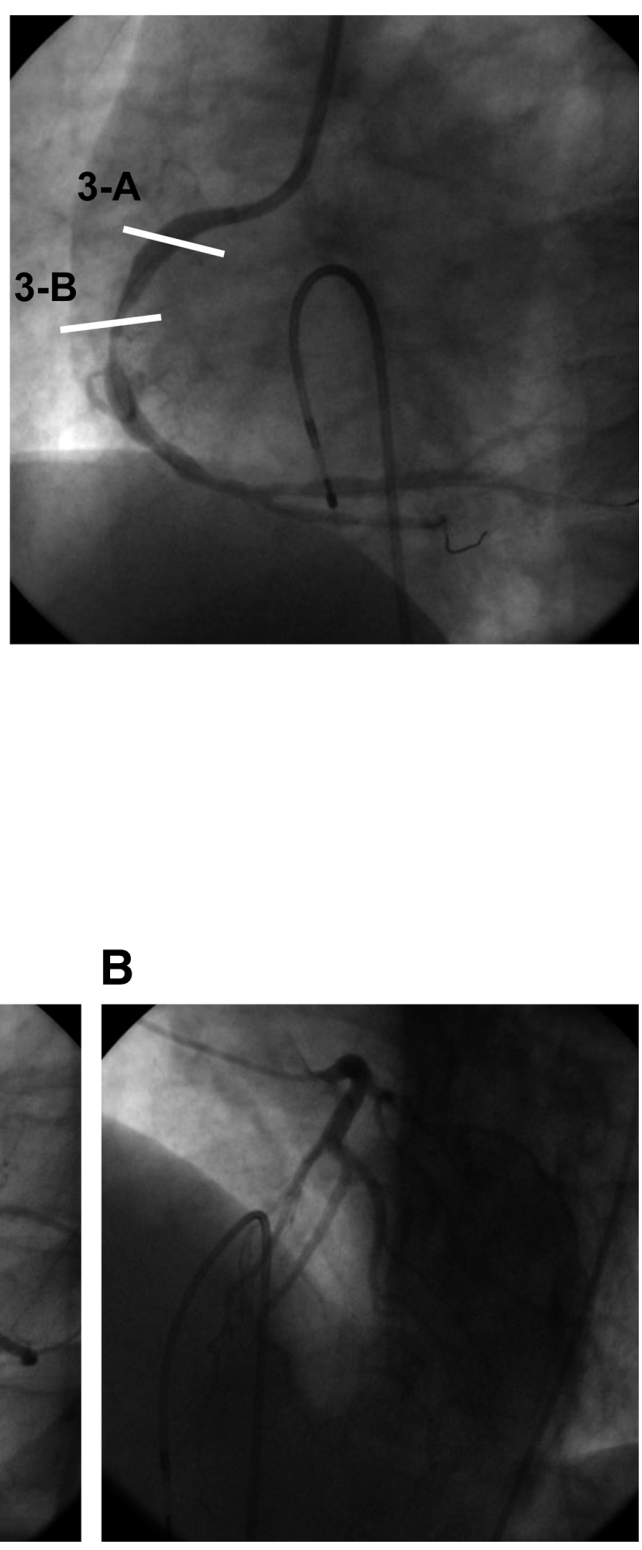

Fig 3. Intravascular ultrasound reveals negative arterial remodeling at the site of coronary spasm in the right coronary artery (RCA). (A) Normal segment of the RCA, showing lumen cross-sectional area (CSA) (arrow). (B) Stenosis of the RCA, showing lumen CSA (arrow).

Fig 4. Angiography after administration of nicorandil shows almost normal coronary arteries.
Fig 5. (A) Coronary angiography shows total occlusion of the posterior lateral branch and diffuse stenosis of the posterior descending branch (arrow). (B) Angiography after administration of nicorandil shows almost normal coronary arteries. 
patients ${ }^{8}$ and is usually alleviated by intracoronary ISDN, although the underlying mechanism remains unclear. Coronary vasomotor tone is maintained by the balance between contraction and relaxation of vascular smooth muscle. If vasomotor tone is too high, coronary artery spasm may occur. Coronary vasomotor tone is regulated by nitric oxide (NO) produced by vascular endothelial cells, but it has been shown that excessive contraction of coronary artery smooth muscle leads to abnormal vasomotor tone, with the vascular endothelium not being important. ${ }^{10}$ Accordingly, the pathogenesis remains unclear.

If endothelial cells are injured, the blood level of NO decreases. Administration of ISDN supplies NO, but was not effective in the present patient, suggesting that damage to vascular smooth muscle was involved. On first admission, she developed cardiogenic shock and we were unable to administer verapamil!1 On the second admission, verapamil was administered intravenously $1^{12}$ but the coronary vasospasm was not improved. However, intracoronary nicorandil was effective on both occasions.

We administered only $1 \mathrm{mg}$ of ISDN because the patient progressed to cardiogenic shock with hemodynamic collapse. Ejima et al ${ }^{13}$ reported that nicorandil $1.0 \mathrm{mg}$ and ISDN $1.0 \mathrm{mg}$ exhibited an almost equivalent coronary dilating effect, and ISDN had the added disadvantage of causing hypotension with resultant tachycardia. They reported that intracoronary injection of ISDN $1.0 \mathrm{mg}$ did not cause arrhythmia or hypotension. Inoue et al reported that $2 \mathrm{mg}$ of nicorandil is the optimal dose ${ }^{14}$ and there have been no previous reports on the effect of intracoronary administration of $3 \mathrm{mg}$ nicorandil.

Nicorandil acts as both a nitrate and a potassium-channel opener. It activates cytoplasmic guanylate cyclase (GC), which leads to an increase in cellular levels of cyclic guanosine monophosphate (cGMP) and a reduction in cytosolic calcium, with subsequent relaxation of vascular smooth muscle ${ }^{15}$ As a potassium-channel opener, nicorandil increases the efflux of $\mathrm{K}^{+}$from cells, which causes hyperpolarization of the cell membrane and indirectly suppresses opening of the voltage-dependent $\mathrm{Ca}^{2+}$ channels, resulting in vasodilation! ${ }^{16}$ In addition, nicorandil increases the cGMP levels in a NO-independent manner via direct activation of GC!7 In the present case symptoms could not relieved by ISDN, but were by nicorandil, so we consider that there was either a direct effect of nicorandil or that it acted via the potassium channels, which led to the improvement of coronary artery spasm.

The vascular potassium channel is an assembly of Kir 6.1 and SUR 2B!8 Both Kir6.1 and SUR2-null mice show an identical phenotype of spontaneous coronary artery spasm and resultant sudden death, resembling Prinzmental angina in humans!9,20 These reports have pointed out an important possibility that the potassium channel may play a critical role in the pathogenesis of coronary vasospasm.

Noguchi et al reported that refractory vasospasm could be relieved by intracoronary injection of $1 \mathrm{mg}$ nicorandil, so we administered that dose initially, but the patient's blood pressure did not fall, and her ECG showed a normal range of QT length. Therefore, we administered another $1 \mathrm{mg}$ of nicorandil (total $2 \mathrm{mg}$ of nicorandil). Nicorandil was effective for vasospasm that was not relieved by intracoronary ISDN or intravenous verapamil, presumably because of its action on potassium channels.

In conclusion, coronary vasospasm affecting multiple vessels caused hemodynamic collapse in the present patient. ISDN and calcium-channel blockers could not relieve it, but the potassium-channel opener nicorandil did. Therefore, nicorandil should be considered as a treatment option when vasospasm occurs in multiple vessels and there is hemodynamic collapse, a situation in which physicians hesitate to administer ISDN and calcium-channel blockers.

\section{References}

1. Suzuki N, Hiasa Y, Miyazaki S, Tomokane T, Ogura R, Miyajima H, et al. Acute myocardial infarction caused by simultaneous occlusion of the right coronary artery and the left anterior descending coronary artery probably due to coronary spasm. J Cardiol 2005; 45: 213-217.

2. Vincent GM, Anderson JF, Marshall HM. Coronary spasm producing coronary thrombosis and myocardial infarction. $N$ Engl J Med 1983; 309: 220-223.

3. Horimoto M, Takenaka T, Igarashi K, Fujiwara M, Batra S. Coronary spasm as a cause of coronary thrombosis and myocardial infarction. Jpn Heart J 1993; 34: 627-631.

4. Taniguchi Y, Nakamura M, Suzuki T, Suzuki T, Aoki H, Fukami KI. A case of acute myocardial infarction: Intracoronary thrombus formation at a previously provoked vasospasm site. Jpn Heart J 2000; 41: 761-766.

5. Saito H, Itoh T, Itoh M, Kanaya Y, Suzuki T, Hiramori K. Simultaneous multivessel coronary spasm causing acute myocardial infarction. Angiology 2007; 58: 112-117.

6. Noguchi T, Nonogi H, Yasuda S, Daikoku S, Morii I, Itoh A, et al. Refractory coronary spasm relieved by intracoronary administration of nicorandil. Circ J 2000; 64: 396-398.

7. Hong MK, Park SW, Lee CW, Ko JY, Kang DH, Song JK, et al. Intravascular ultrasound findings of negative arterial remodeling at sites of focal coronary spasm in patients with vasospastic angina. Am Heart J 2000; 140: 395-401.

8. Miwa K, Fujita M, Sasayama S. Recent insights into the mechanisms, predisposing factors, and racial differences of coronary vasospasm. Heart Vessels 2005; 20: 1-7.

9. Furchgott R, Zawadzki JV. The obligatory role of endothelial cells in the relaxation of artery smooth muscle by acetylcholine. Nature 1980; 288: $377-376$

10. Yasue H, Omote S, Takizawa A, Nagao M. Coronary artery spasm in ischemic heart disease and its pathogenesis. Circ Res 1983; 52: S147-S152.

11. David G. Intracoronary verapamil for reversal of refractory coronary vasospasm during percutaneous transluminal coronary angioplasty. $J$ Am Coll Cardiol 1988; 12: 1377-1381.

12. Gaetano L, Attino M. Coronary artery spasm. Curr Treatment Options Cardiovasc Med 2000; 2: 83-90.

13. Ejima J, Kaneda K, Moriyama H, Ohmura I, Maruyama T, Kaji Y, et al. Coronary dilating effects of intracoronary nicorandil: Comparison with isosorbide dinitrate. Jpn Heart J 1995; 36: 699-707.

14. Inoue F, Hashimoto T, Fujimoto S, Uemura S, Kawamoto A, Dohi K. Estimation of coronary flow reserve by intracoronary administration of nicorandil. Heart Vessels 1998; 13: 229-236.

15. Simpson D, Wellington K. Nicorandil: A review of its use in the management of stable angina pectoris, including high-risk patients. Drugs 2004; 64: 1941-1955.

16. Miura T, Miki T. ATP-sensitive $\mathrm{K}^{+}$channel openers: Old drugs with new clinical benefits for the heart. Curr Vasc Pharmacol 2003; 1: $251-258$.

17. Minamiyama Y, Takemura S, Hai S, Suehiro S, Okada S, Funae Y. Nicorandil elevates tissue cGMP levels in a nitric-oxide-independent manner. J Pharmacol Sci 2007; 103: 33-39.

18. Seino S, Miki T. Physiological and pathophysiological roles of ATPsensitive $\mathrm{K}^{+}$channels. Prog Biophys Mol Biol 2003; 81: 133-176.

19. Miki T, Suzuki M, Shibasaki T, Uemura H, Sato T, Yamaguchi K, et al. Mouse model of Prinzmetal angina by distribution of the inward rectifier Kir6.1. Nature 2002; 8: 466-472.

20. Kakkar R, Ye B, Stoller DA, Smelly M, Shin N, Galles K, et al. Spontaneous coronary vasospasm in KATP mutant mice arises from a smooth muscle-extrinsic process. Circ Res 2006; 98: 682-689. 